EPJ Web of Conferences 85, 02006 (2015)

DOI: $10.1051 /$ epjconf/ 20158502006

(C) Owned by the authors, published by EDP Sciences, 2015

\title{
Gluon contribution to the Sivers effect. COMPASS results on deuteron target.
}

\author{
Adam Szabelski ${ }^{1, a}$ on behalf of the COMPASS Collaboration \\ ${ }^{1}$ National Centre for Nuclear Research, Warsaw
}

\begin{abstract}
Sivers effect for gluons is connected to gluon orbital angular momentum which may be the missing part of the nucleon spin puzzle. We present a method of extraction of Sivers effect for gluons from COMPASS SIDIS data on transversely polarised target. In order to access the Sivers effect for gluons photon-gluon fusion (PGF) process is used. To enhance the fraction of PGF in the sample high- $p_{T}$ hadron pair events are selected. The method is based on a assumption that there are 3 processes contributing to the muon-nucleon scattering: PGF, leading process and QCD Compton process. Then one performs a weighting procedure which enables to extract the asymmetries for the 3 contributing processes simultaneously. In order to do that a neural network trained by a Monte Carlo to assign to each event 3 probabilities corresponding to the 3 processes is needed. Finaly we show results of Sivers effect for gluons extraction on COMPASS data with transversely polarised deuteron target. $\mathbf{A}_{\mathbf{P G F}}^{\sin \phi_{2 \mathrm{~h}}-\phi_{\mathbf{S}}}=\mathbf{- 0 . 1 4} \pm \mathbf{0 . 1 5}$ (stat.) at $\left\langle x_{G}\right\rangle=0.126$.
\end{abstract}

\section{Introduction}

In the paper we present the extraction of the Sivers effect for gluons. In section 2 the analysis method enabling us to extract Sivers effect from COMPASS data is presented. Moreover the method enables us to separate the Sivers effect comming from the Photon-Gluon Fusion (PGF) and quark-originated processes in leading order. Then, in Section 3 the agreement between MC and data is presented. MC is used to train a Neural Network and we demonstrate the validation of this training. In Section 4 a Monte Carlobased example of application of our method is shown. In Section 5 data selection for COMPASS data taken on transversaly polarised deuteron target is presented. Finally, in Section 6 the results are shown. In Section 7 the main source of the systematic error that is due to the choice of MC is discussed.

\section{Determination of Sivers asymmetry for gluons for $Q^{2}>1(\mathrm{GeV} / c)^{2}$}

\subsection{The measurement of Sivers asymmetry}

The lepton-proton scattering cross-section containts 8 transverse momentum dependent modulation in the azimuthal angles including Sivers modulation, Ref. [4].

The transverse single spin SIDIS asymmetry is given by:

$$
A_{T}^{h} \sim \frac{d^{6} \sigma^{\uparrow}-d^{6} \sigma^{\downarrow}}{d^{6} \sigma^{\uparrow}+d^{6} \sigma^{\downarrow}} .
$$

SIDIS measurement with a transversely polarised target allows to disentangle all 8 asymmetries (including the Sivers

\footnotetext{
ae-mail: adam.szabelski@cern.ch
}

asymmetry) using one data set. Throughout this paper only Sivers asymmetry will be mentioned.

In the following the set of all kinematic variables is denoted by a vector $\vec{x}$ :

$$
\vec{x}=\left(\vec{p}_{i}, \overrightarrow{v_{i}}, Q^{2}, y, \ldots\right) .
$$

The number of observed interactions in target cell $c=$ $u, d, u^{\prime}, d^{\prime}$ can be written as:

$$
n_{c}(\vec{x})=\alpha_{c}(\vec{x})\left(1+\beta_{c}(\vec{x}) A_{U T}^{\sin \left(\phi_{2 h}-\phi_{s}\right)}(\vec{x})\right),
$$

where $u, d$ are the upstream and downstream target cells before reversal of the target polarisation and $u^{\prime}, d^{\prime}$ denote the cells after reversing the polarisation of the target. Moreover

$$
\begin{gathered}
\alpha_{c}(\vec{x})=a_{c} \Phi n_{c} \sigma, \\
\beta_{c}(\vec{x})=P_{T} f \sin \left(\phi_{2 h}-\phi_{s}\right),
\end{gathered}
$$

where $\phi_{2 h}$ is the azimuthal angle of the vector sum of the leading and next-to-leading hadron momenta. Here the leading hadron is the hadron with the largest transverse momentum. The dilution factor $f$ is defined by the percentage of polarisable material in the target.

By applying the weighted method Eq. (3) can be weighted with a weighting function $\omega(\vec{x})$ :

$$
\begin{aligned}
p_{c} & :=\int \omega(\vec{x}) n_{c}(\vec{x}) d \vec{x}=\int \omega(\vec{x}) \alpha_{c}(\vec{x}) d \vec{x} \\
& +\int \omega(\vec{x}) \alpha_{c}(\vec{x}) \beta_{c}(\vec{x}) A_{U T}^{\sin \left(\phi_{2 h}-\phi_{s}\right)}(\vec{x}) d \vec{x} \approx \sum_{i=1}^{N_{c}} \omega_{i}
\end{aligned}
$$

where the sum $\sum_{i=1}^{N_{c}} \omega_{i}$ runs over all events with the primary vertex in cell $c$. Then it can be rewritten in the following 
form:

$$
\sum_{i=1}^{N_{c}} \omega_{i}=\tilde{\alpha}_{c}\left(1+\left\{\beta_{c}\right\}_{\omega}\left\{A_{U T}^{\sin \left(\phi_{2 h}-\phi_{s}\right)}\right\}_{\omega \beta_{c}}\right)
$$

where

$$
\begin{gathered}
\tilde{\alpha}_{c}=\int \omega(\vec{x}) \alpha_{c}(\vec{x}) d \vec{x} \\
\left\{A_{U T}^{\sin \left(\phi_{2 h}-\phi_{s}\right)}\right\}_{\omega \beta_{c}}=\frac{\int A_{U T}^{\sin \left(\phi_{2 h}-\phi_{s}\right)}(\vec{x}) \omega(\vec{x}) \beta_{c} \alpha_{c}(\vec{x}) d \vec{x}}{\int \omega(\vec{x}) \beta_{c} \alpha_{c}(\vec{x}) d \vec{x}} \\
\{\beta\}_{\omega}=\frac{\int \beta(\vec{x}) \omega(\vec{x}) \alpha_{c}(\vec{x}) d \vec{x}}{\int \omega(\vec{x}) \alpha_{c}(\vec{x}) d \vec{x}} \approx \frac{\sum_{i} \beta_{i} \omega_{i}}{\sum_{i} \omega_{i}} .
\end{gathered}
$$

Here index $c\left(=u, u^{\prime}, d, d^{\prime}\right)$ stands for the target cell. Assuming a linear dependence of $A_{U T}^{\sin \left(\phi_{2 h}-\phi_{s}\right)}$ as a function of $x$, Sivers asymmetry can be written as $A_{U T}^{\sin \left(\phi_{2 h}-\phi_{s}\right)}(x)=$ $a(x-\langle x\rangle)+A_{U T}^{\sin \left(\phi_{2 h}-\phi_{s}\right)}(\langle x\rangle)$. If $\langle x\rangle_{\omega \beta_{u}} \approx\langle x\rangle_{\omega \beta_{d}} \approx\langle x\rangle_{\omega \beta_{u^{\prime}}} \approx$ $\langle x\rangle_{\omega \beta_{d^{\prime}}} \equiv\langle x\rangle$ one can simplify Eq. (7) and write:

$$
\sum_{i=1}^{N_{c}} \omega_{i}=\tilde{\alpha}_{c}\left(1+\{\beta\}_{\omega} A_{U T}^{\sin \left(\phi_{2 h}-\phi_{s}\right)}(\langle x\rangle)\right)
$$

Using the assumption for integrated acceptances:

$$
\frac{\tilde{\alpha_{u}} \tilde{\alpha_{d^{\prime}}}}{\tilde{\alpha_{d}} \tilde{\alpha_{u^{\prime}}}}=1
$$

the 4 equations (one for each target configuration) with 5 unknowns ( $\tilde{\alpha}_{c}$ and $A_{U T}^{\sin \left(\phi_{2 h}-\phi_{s}\right)}$ ) can be reduced to 4 equations with 4 unknowns and the set of equations becomes solvable.

So far the function $\omega(\vec{x})$ is arbitrary, but it can be shown that the best choice, taking into account statistic and systematic error of the extracted asymmetry, is:

$$
\omega(\vec{x})=\frac{\beta(\vec{x})}{P_{T}}=f \sin \left(\phi_{2 h}-\phi_{s}\right) .
$$

\subsection{Extraction of Sivers asymmetry on gluons from measured asymmetry}

The Sivers asymmetry for the production of two high- $p_{T}$ hadrons in the large $Q^{2}$ regime can be expressed as follows:

$$
\begin{aligned}
A_{U T}^{\sin \left(\phi_{2 h}-\phi_{s}\right)} & =R_{P G F} A_{P G F}^{\sin \left(\phi_{2 h}-\phi_{s}\right)}\left(\left\langle x_{G}\right\rangle\right)+R_{L P} A_{L P}^{\sin \left(\phi_{2 h}-\phi_{s}\right)}\left(\left\langle x_{B j}\right\rangle\right) \\
& +R_{Q D C D} A_{Q C D C}^{\sin \left(\phi_{2 h}-\phi_{s}\right)}\left(\left\langle x_{C}\right\rangle\right)
\end{aligned}
$$

Here the coefficients $R$ are the fractions of the three QCD leading order subprocesses with labels LP , QCDC and PGF that refer to leading process $\gamma q \rightarrow q$, QCD Compton $\gamma q \rightarrow q g$, photon-gluon fusion $\gamma g \rightarrow q \bar{q}$. The method of extracting $A_{P G F}^{\sin \left(\phi_{2 h}-\phi_{s}\right)}, A_{L P}^{\sin \left(\phi_{2 h}-\phi_{s}\right)}, A_{Q C D C}^{\sin \left(\phi_{2 h}-\phi_{s}\right)}$ combines approaches of two COMPASS analysis: the high- $p_{T}$ hadron pair analysis with longitudinally polarized target for extracting gluon polarisation $\Delta g / g$, [1] and the open charm analysis, [2] for separtion of the signal (PGF process) and background (LP and QCDC). It was used in all- $p_{T}$ hadrons analysis for longitudinally polarized target at COMPASS for extracting $\Delta g / g$, [3]. After substituting Eq. (14) into Eq. (3) one can weight the outcome with 3 weights:

$$
\begin{gathered}
\omega_{P G F} \equiv \omega^{G}=R_{P G F} f \sin \left(\phi_{2 h}-\phi_{s}\right)=\frac{\beta^{G}}{P_{T}} \\
\omega_{L P} \equiv \omega^{L}=R_{L P} f \sin \left(\phi_{2 h}-\phi_{s}\right)=\frac{\beta^{L}}{P_{T}}, \\
\omega_{Q C D C} \equiv \omega^{C}=R_{Q C D C} f \sin \left(\phi_{2 h}-\phi_{s}\right)=\frac{\beta^{C}}{P_{T}} .
\end{gathered}
$$

Using these weights we can write 12 equations:

$$
\begin{aligned}
p_{c}^{j} & =\sum_{i=1}^{N_{c}} \omega_{i}^{j}=\tilde{\alpha}_{c}^{j}\left(1+\left\{\beta_{c}^{G}\right\}_{\omega^{j}} A_{P G F}^{\sin \left(\phi_{2 h}-\phi_{s}\right)}\left(\left\langle x_{G}\right\rangle\right)\right. \\
& \left.+\left\{\beta_{c}^{L}\right\}_{\omega^{j}} A_{L P}^{\sin \left(\phi_{2 h}-\phi_{s}\right)}\left(\left\langle x_{B j}\right\rangle\right)+\left\{\beta_{c}^{C}\right\}_{\omega^{j}} A_{Q C D C}^{\sin \left(\phi_{2 h}-\phi_{s}\right)}\left(\left\langle x_{C}\right\rangle\right)\right) \\
& =\tilde{\alpha}_{c}^{j}\left(1+A_{P G F}\left\{\beta^{G}\right\}_{\omega^{j}}+A_{L P}\left\{\beta^{L}\right\}_{\omega^{j}}+A_{Q C D C}\left\{\beta^{C}\right\}_{\omega^{j}}\right) .
\end{aligned}
$$

Here $j=P G F, L P, Q C D C$ and linear dependence of the asymmetries on $x$ is assumed (see App. A). This way we get 12 equations for 15 unknowns ( 3 asymmetries and 12 integrated acceptances). Again the assumption:

$$
\frac{\tilde{\alpha}_{u}^{j} \tilde{\alpha}_{d^{\prime}}^{j}}{\tilde{\alpha}_{d}^{j} \tilde{\alpha}_{u^{\prime}}^{j}}=1
$$

limits the number of unknowns to 12 .

We can now construct two vectors:

$$
\begin{aligned}
& \overrightarrow{N_{o b s}}=\left(\sum_{i=0}^{N_{u}} \omega_{i}^{G}, \ldots, \sum_{i=0}^{N_{d^{\prime}}} \omega_{i}^{C}\right), \\
& \overrightarrow{N_{\text {exp }}}=\left(N_{\text {exp }, G}^{u}, \ldots, N_{\text {exp }, C}^{d^{\prime}}\right),
\end{aligned}
$$

where the expected number of events is given by:

$$
N_{\text {exp }, j}^{c}=\tilde{\alpha}_{c}^{j}\left(1+A_{P G F}\left\{\beta^{G}\right\}_{\omega^{j}}+A_{L P}\left\{\beta^{L}\right\}_{\omega^{j}}+A_{Q C D C}\left\{\beta^{C}\right\}_{\omega^{j}}\right) .
$$

The asymetries $\left(A_{P G F}, A_{L P}, A_{Q C D C}\right)$ can be obtained by minimizing $\chi^{2}$ :

$$
\chi^{2}=\left(\overrightarrow{N_{e x p}}-\overrightarrow{N_{o b s}}\right)^{T} \operatorname{Cov}^{-1}\left(\overrightarrow{N_{e x p}}-\overrightarrow{N_{o b s}}\right)
$$

The covariance matrix is defined as $\operatorname{Cov}\left(p_{x}, p_{y}\right) \approx$ $\sum_{N_{c}} \omega_{x} \omega_{y}$.

\subsection{Binning in the Sivers angle}

The value of $\sum_{i} \omega=\sum_{i} R_{i} f_{i} \sin \left(\phi_{h}^{i}-\phi_{S}^{i}\right)$ is close to 0 and the value of $\{\beta\}_{\omega} \approx\left(\sum_{i} \beta_{i} \omega_{i}\right) /\left(\sum_{i} \omega_{i}\right)$ becomes large what makes difficult to converge the fit. The solution is to make two $\phi_{h}-\phi_{S}$ bins: $(0, \pi),(\pi, 2 \pi)$. Then one can make a simultaneous fit in the two bins with a constraint $A\left(1 s t \_b i n\right)=A\left(2 n d \_b i n\right)$. 


\section{Monte Carlo simulation and Neural Network training}

The Monte-Carlo (MC) simulations included a generation the physics process, then the propagation of generated particles through the COMPASS spectrometer and the reconstruction of MC events. The physical processes were simulated using LEPTO generator with COMPASS tuning. The MC tuning was the same as in the high- $p_{T}$ analysis for gluon polarisation determination and it is described in detail in Ref. [1]. The only difference in the detector setup is the transverse direction of the target spin. In this section the agreement between MC and real data is presented and the validation of the neural network training is shown.

\subsection{Comparison of MC with real data}

The comparison of MC and real data for distribution (normalized to the number of entries of 3 inclusive kinematic variables $\left(x_{B j}, Q^{2}, y\right)$ and 5 hadronic kinematic variables $\left(p_{T 1}, p_{T 2}, p_{L 1}, p_{L 2}, \sum p_{T}^{2}=p_{T 1}^{2}+p_{T 2}^{2}\right)$ is presented in Fig. 1. From this comparison it can be concluded that the agreement between MC simulation and real data is reasonably good.

\subsection{Neural Network training}

For the neural network (NN) training the MC simulated data was used. As an input a vector of 6 kinematic variables: $p_{T 1}, p_{T 2}, p_{L 1}, p_{L 2}, Q^{2}, x_{B j}$ has been applied. As a target (the values which the $\mathrm{NN}$ tries to reach during the training) the 2 -dimensional vector $(t 1, t 2)$ given by the following:

$$
\begin{aligned}
& P G F: t 1=0 ; \quad t 2=0, \\
& L P: t 1=0.5 ; \quad t 2=\sqrt{3} / 2, \\
& Q C D C: t 1=1 ; \quad t 2=0
\end{aligned}
$$

is applied.

The data set on which the neural network is trained is randomly divided into two parts: the "training set" and the "testing set". The network is trained using the "training set" while the "testing set" is used to monitor the training procedure. When the objective functions obtained from the two sets diverge too much, the training is stopped. The NN was trained using the backpropagation algorithm, [6]. As a result of the training we get a network that assigns to each event that is characterized by the 6 input kinematic variables a 2-dimensional vector $(o 1, o 2)$ from which the process fractions can be obtained in the following way:

$$
\begin{aligned}
R_{P G F} & =1-o 1-1 / \sqrt{3} o 2, \\
R_{L P} & =2 / \sqrt{3} o 2 \\
R_{Q C D C} & =o 1-1 / \sqrt{3} o 2 .
\end{aligned}
$$

To validate the NN training a following test is perform. The MC sample is randomly divided into two equal sets. Each set is divided into bins of $P_{N N}$, the process fraction according to NN output. In each bin the true fractions $P_{M C}=N_{\text {process }} / N_{\text {all }}$ are calculated for each process. Exemplary results are shown in Fig. 2. On the top pannels the
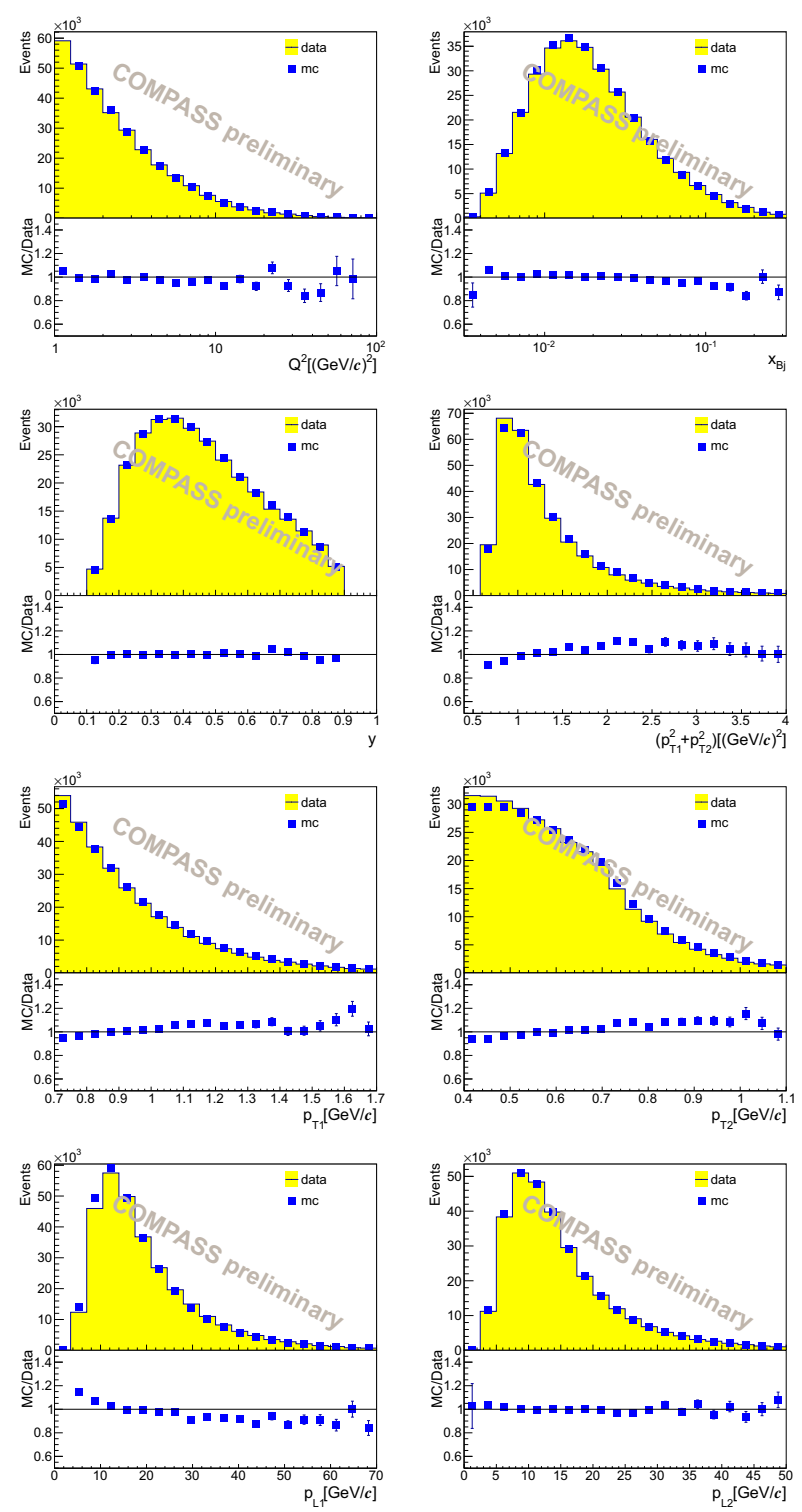

Figure 1. The comparison between Monte-Carlo and real data for different kinematic variables.

points represent the true $\mathrm{MC}$ process fraction $\left(P_{M C}\right)$ as the function of $P_{N N}$. The line shows the expexted dependence. In the bottom pannels the difference $P_{M C}-P_{N N}$ as a function of $P_{N N}$ is evaluated. The results are in a reasonable agreement.

\section{Validation of the method using Monte Carlo}

To validate the method we generate MC events. Each event is tagged with the label of the process (LP, QCDC, PGF) which was used to generate given event. Then every event is weighted by $1+A_{i}^{S I M} \sin \left(\phi_{2 h}-\phi_{S}\right)$, where $i$ labels the process which is known in MC. We put the simulated asymmetries $\left(A_{P G F}^{S I M}, A_{Q C D C}^{S I M}, A_{L P}^{S I M}\right)$ to constant values. To such weighted MC sample we apply the described method of asymmetry extraction expecting to get $A_{i}^{\text {extracted }} \simeq A_{i}^{S I M}$. The results are shown in Fig. 3 . The red 

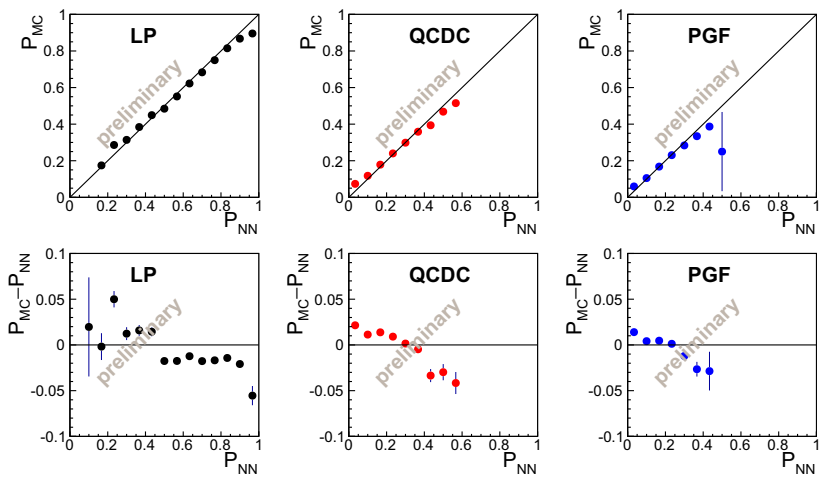

Figure 2. Neural network validation. Here $P_{N N}$ is the fraction of the process given by the NN and $P_{M C}$ is the true fraction of each process from MC in a given $P_{N N}$ bin.

\section{Sivers Asymmetry}
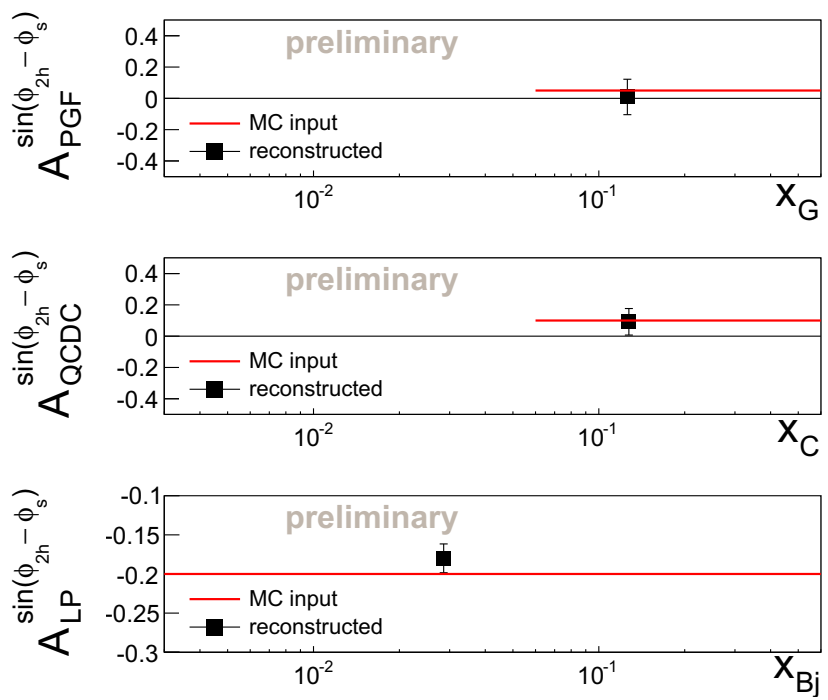

Figure 3. Validation of the analysis method. $A_{P G F}^{S I M}=$ $0.05, A_{Q C D C}^{S I M}=0.1, A_{L P}^{S I M}=-0.2$

lines indicate the simulated values and the points represent the extracted ones. From this results we conclude that the method enables to distuinguish between asymmetries of different processes.

\section{Data sample and event selection}

The data sample used includes data taken in 2003 and 2004 with transversely polarised deuteron target.

\subsection{Polarisation of the target}

The target was constructed of two oppositely polarised cells ("up" cell and "down" cell). The polarisation in both cells where inverted every several days.

The extrapolated track of incoming muon, $\mu$, is required to cross both target cells and the primary vertex to be inside the nominal cell volumes.

\subsection{Particle identification}

The scattered muon candidate, $\mu^{\prime}$, is required to be very penetrating and therefore a cut $X / X_{0}>30$ is used on the number of traversed radiation lengths. Here $X$ is the measured length of the track of the particle, $X_{0}$ the avarage radiation length of the materials through which the particle passed. It is also required to pass through the active area of the trigger hodoscopes that have fired for the considered event. The event is also rejected when there is a high momentum particle passing through the absorber's hole. Additionally there is a requirement for the reduced $\chi^{2}$ of the track fit $\left(\chi^{2} / n d f<10\right)$. The scattered muon track is required to have at least one hit in detectors upstream the SM1 magnet.

In case of more than one $\mu^{\prime}$ candidate in the event, the event is rejected. Moreover the $\mu^{\prime}$ track must not cross yoke of the second magnet SM2.

Two particles coming from the primary vertex with the largest $p_{T}$ with respect to the virtual photon $q$ are considered as hadron candidates. First of all, both of them should not be identified as muons. Therefore the number of radiation lengths corresponding to their tracks should be small $\left(X / X_{0}<10\right)$. In order to avoid tracks reconstructed in the SM1 fringe field, we also require for a track of hadron candidate to have one hit before the SM1 magnet and one hit after SM1. Finally there is a cut on the reduced $\chi^{2}$ of the track fit $\left(\chi^{2} / n d f<10\right)$.

\subsection{Kinematic cuts}

There are two major reasons for choosing the high$p_{T}$ sample. First, with this choice the fraction of PGF in the sample is enhanced. Secondly it improves the correlation between the azimuthal angle of the gluon momentum and the azimuthal angle of the reconstructed sum of two leading hadron momenta.

The following cuts on inclusive kinematic variables were applied: $Q^{2}>1(\mathrm{GeV} / c)^{2}$ to select events in the perturbative region, $0.003<x_{B j}<0.7,0.1<y<0.9$ and $W>5 \mathrm{GeV} / c^{2}$.

For further sellection following hadronic cuts were used: the high- $p_{T}$ cut $p_{T 1}>0.7 \mathrm{GeV} / c, p_{T 2}>0.4 \mathrm{GeV} / c$, which ensures that PGF fraction in the sample is enhanced and that the correlation between the azimuthal angle of the gluon and the azimuthal angle of the vector sum of two hadrons is stronger. The cuts $z_{1}>0.1$ and $z_{2}>0.1$ are used to select current fragmentation region and the cut $z_{1}+z_{2}<0.9$ is applied to reject events from exclusive production.

\section{Results}

\subsection{Final results}

The Sivers asymmetry values are calculated in three periods of data taking. Each period consists of two data sets 
with opposite target polarisation. The final result is obtained according to the following formula:

$$
A_{\text {final }}=\frac{\sum_{i=1}^{3} \frac{A_{i}}{\sigma_{i}^{2}}}{\sum_{i=1}^{3} \frac{1}{\sigma_{i}^{2}}}, \quad \sigma_{\text {final }}^{2}=\frac{1}{\sum_{i=1}^{3} \frac{1}{\sigma_{i}^{2}}}
$$

and is presented in Fig. 4. The result for the Sivers asym-

\section{Sivers Asymmetry}
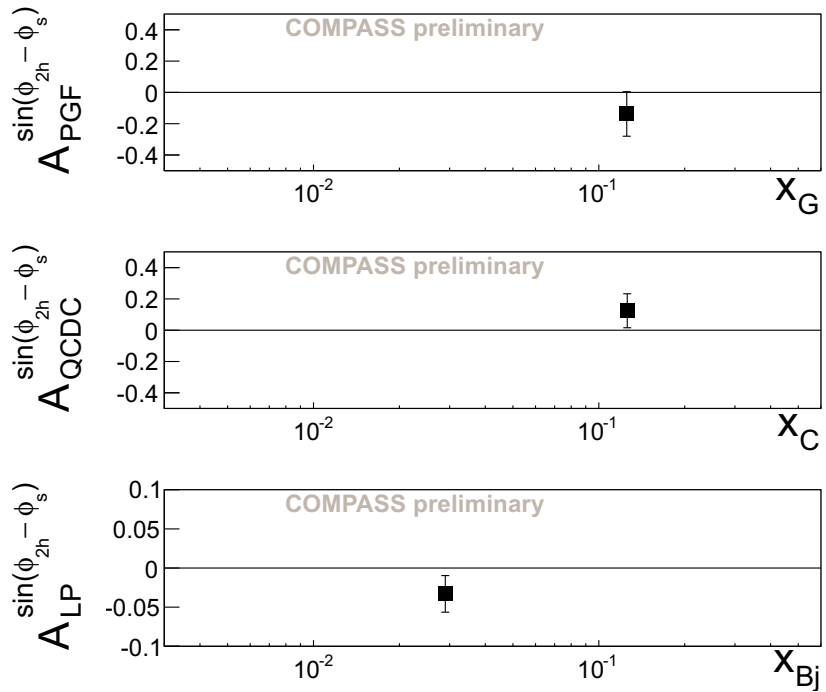

Figure 4. Final Sivers asymmetries for different QCD processes for 2003 and 2004 deuteron data.

metry for gluons is $\mathrm{A}_{\mathbf{P G F}}^{\sin \left(\phi_{2 \mathrm{~h}}-\phi_{\mathbf{S}}\right)}=-\mathbf{0 . 1 4} \pm \mathbf{0 . 1 5}$ (stat.) at $\left\langle x_{G}\right\rangle=0.126$. The result for the Sivers asymmetry for the leading process $A_{L P}^{\sin \left(\phi_{2 h}-\phi_{S}\right)}=-0.033 \pm 0.024$ (stat.) at $\left\langle x_{B j}\right\rangle=0.028$ and for the QCD-Compton: $A_{Q C D C}^{\sin \left(\phi_{2 h}-\phi_{S}\right)}=$ $0.12 \pm 0.11$ (stat.) at $\left\langle x_{C}\right\rangle=0.137$.

It should be added that the $A_{L P}^{\sin \left(\phi_{2 h}-\phi_{S}\right)}$ is not be expected to be the same as the standard Sivers asymmetry measured by COMPASS, Ref. [5]. This result is obtained on a high- $p_{T}$ hadron pair subsample and the correlation between parton azimuthal angles and reconstructed hadron azimuthal angles is different what leads to different measured asymmetries.

\section{Systematic studies}

\subsection{Systematic error due to MC}

Several different MC samples were produced for systematic studies, as in Ref. [1] differing by the choice of PDF model (CTEQ or MSTW), LEPTO tuning (default or tuned to COMPASS data), parton shower on or off, $F_{L}$ from LEPTO or from the $R=\sigma_{L} / \sigma_{T}$. Namely:

1. LEPTO DEF. tuning, parton shower ON, $\mathrm{PDF}=\mathrm{CTEQ5L}$;

2. LEPTO DEF. tuning, parton shower OFF, $\mathrm{PDF}=\mathrm{MSTW08}$;
3. LEPTO DEF. tuning, parton shower ON, $\mathrm{PDF}=\mathrm{MSTW} 08$;

4. LEPTO COMPASS tuning, parton shower ON, $\mathrm{PDF}=\mathrm{CTEQ5L}$;

5. LEPTO COMPASS tuning, parton shower OFF, $\mathrm{PDF}=\mathrm{MSTW08}$

6. LEPTO COMPASS tuning, parton shower ON, $\mathrm{PDF}=\mathrm{MSTW} 08$, NO FL;

7. LEPTO COMPASS tuning, parton shower ON, $\mathrm{PDF}=\mathrm{MSTW08}$.

For each of them aymmetry $A_{P G F}$ has been calculated in the same way as the final results and all of them were used in the systematics studies. The results are presented in Fig. 5. Red colour indicates the MC used to obtain the final result. For the systematic error that is due to $\mathrm{MC}$ tuning

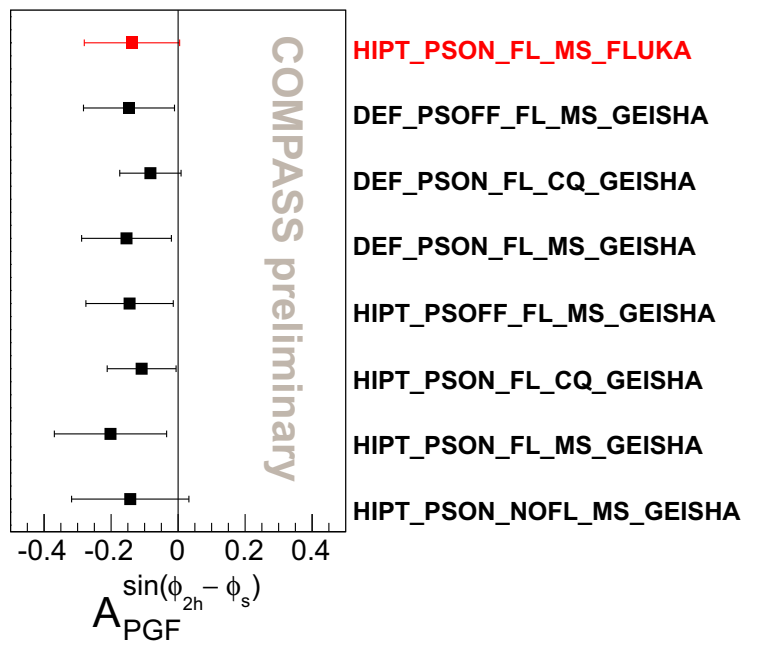

Figure 5. $A_{P G F}$ for different $\mathrm{MC}$ simulations.

the half of the maximum difference of obtained results has been chosen, which is 0.06 .

\section{A Approximation of asymmetries by linear dependence on $x$}

After substituting Eq. (14) into Eq. (3)

$$
\begin{aligned}
n_{c}(\vec{x})= & \alpha_{c}(\vec{x})\left(1+\beta_{c}^{G}(\vec{x}) A_{P G F}^{\sin \left(\phi_{2 h}-\phi_{s}\right)}(\vec{x})\right. \\
& \left.+\beta_{c}^{L}(\vec{x}) A_{L P}^{\sin \left(\phi_{2 h}-\phi_{s}\right)}(\vec{x})+\beta_{c}^{C}(\vec{x}) A_{Q C D C}^{\sin \left(\phi_{2 h}-\phi_{s}\right)}(\vec{x})\right)
\end{aligned}
$$

The outcome can be weighted with 3 weights:

$$
\begin{gathered}
\omega_{P G F} \equiv \omega^{G}=R_{P G F} f \sin \left(\phi_{2 h}-\phi_{s}\right)=\frac{\beta^{G}}{P_{T}}, \\
\omega_{L P} \equiv \omega^{L}=R_{L P} f \sin \left(\phi_{2 h}-\phi_{s}\right)=\frac{\beta^{L}}{P_{T}}, \\
\omega_{Q C D C} \equiv \omega^{C}=R_{Q C D C} f \sin \left(\phi_{2 h}-\phi_{s}\right)=\frac{\beta^{C}}{P_{T}} .
\end{gathered}
$$


Using these weights we can write 12 equations:

$$
\begin{aligned}
p_{c}^{j} & =\sum_{i=1}^{N_{c}} \omega_{i}^{j}=\tilde{\alpha}_{c}^{j}\left(1+\left\{\beta_{c}^{G}\right\}_{\omega^{j}}\left\{A_{P G F}^{\sin \left(\phi_{2 h}-\phi_{s}\right)}\right\}_{\omega^{j} \beta_{c}^{G}}\right. \\
& \left.+\left\{\beta_{c}^{L}\right\}_{\omega^{j}}\left\{A_{L P}^{\sin \left(\phi_{2 h}-\phi_{s}\right)}\right\}_{\omega^{j} \beta_{c}^{L}}+\left\{\beta_{c}^{C}\right\}_{\omega^{j}}\left\{A_{Q C D C}^{\sin \left(\phi_{2 h}-\phi_{s}\right)}\right\}_{\omega^{j} \beta_{c}^{C}}\right) .
\end{aligned}
$$

Here $j=G, L, C \equiv P G F, L P, Q C D C$. Asymmetries $A_{j}^{\sin \left(\phi_{2 h}-\phi_{s}\right)}\left(x_{j}\right)$ can be approximated with a linear function, as discussed in Section 2.1. To simplify the Eqs. (27) one needs to have $\left\{x_{j}\right\}_{\omega^{k} \beta_{c}^{j}} \approx\left\{x_{j}\right\}_{\omega^{m} \beta_{b}^{j}} \equiv$ $\left\langle x_{j}\right\rangle \quad j, k, m=G, L, C$ and $c, b$ run over $u, d, u^{\prime}, d^{\prime} 12$ such values for each process can be approximated by $\left\{x_{j}\right\}_{\omega^{k} \beta_{c}^{j}} \approx\left(\sum_{i} \omega_{i}^{k} \beta_{i}^{j} x_{j}^{i}\right) /\left(\sum_{i} \omega_{i}^{k} \beta_{i}^{j}\right)$. Here summation over $i$ is performed for each cell $c$. The results are stored in the Table 1. Taking the average value of $\left\langle x_{P G F}\right\rangle \equiv\left\langle x_{G}\right\rangle$,

\begin{tabular}{|c|c|c|c|c|c|c|c|c|}
\hline & \multicolumn{4}{|c|}{$\omega_{L P}$} & \multicolumn{4}{|c|}{$\omega_{Q C D C}$} \\
\hline & $\mathrm{u}$ & $\mathrm{d}$ & $u^{\prime}$ & d' & $\mathrm{u}$ & d & $\mathrm{u}^{\prime}$ & $\mathrm{d}^{\prime}$ \\
\hline$\overline{\left\langle\left\langle x_{G}\right\rangle\right.}$ & 0.1197 & 0.1245 & 0.1197 & 0.1245 & 0.126 & 0.1317 & \begin{tabular}{l|l}
7 & 0.1259
\end{tabular} & 0.1316 \\
\hline$\left\langle x_{B j}\right\rangle$ & 0.0249 & 0.0324 & 0.0246 & 0.0324 & 0.0313 & 0.0381 & 0.0309 & 0.038 \\
\hline$\left\langle x_{C}\right\rangle$ & 0.1222 & 0.140 & 0.12 & 0.14 & 0.1375 & 0.1568 & 0.137 & 0.1568 \\
\hline & & \multicolumn{4}{|c|}{$\omega_{P G F}$} & \multirow[b]{2}{*}{ RMS } & \multirow[b]{2}{*}{$\langle x\rangle$} & \\
\hline & & $\mathrm{u}$ & $\mathrm{d}$ & u' & $\mathrm{d}^{\prime}$ & & & \\
\hline & $\left\langle x_{G}\right\rangle$ & 0.1231 & 0.1288 & 0.1231 & 0.1287 & 0.0038 & 0.1256 & \\
\hline & $\left\langle x_{B j}\right\rangle$ & 0.0191 & 0.0221 & 0.019 & 0.022 & 0.0065 & 0.0279 & \\
\hline & $\left\langle x_{C}\right\rangle$ & 0.1233 & 0.1408 & 0.1232 & 0.1405 & 0.0118 & 0.1368 & \\
\hline
\end{tabular}
$\left\langle x_{L P}\right\rangle \equiv\left\langle x_{B j}\right\rangle$ and $\left\langle x_{Q C D C}\right\rangle \equiv\left\langle x_{C}\right\rangle$ one can rewrite

Table 1. Weighted averages of $\mathrm{x}$ for 3 contributing processes. equations (27) as follows:

$$
\begin{aligned}
p_{c}^{j} & =\sum_{i=1}^{N_{c}} \omega_{i}^{j}=\tilde{\alpha}_{c}^{j}\left(1+\left\{\beta_{c}^{G}\right\}_{\omega^{j}} A_{P G F}^{\sin \left(\phi_{2 h}-\phi_{s}\right)}\left(\left\langle x_{G}\right\rangle\right)\right. \\
& \left.+\left\{\beta_{c}^{L}\right\}_{\omega^{j}} A_{L P}^{\sin \left(\phi_{2 h}-\phi_{s}\right)}\left(\left\langle x_{B j}\right\rangle\right)+\left\{\beta_{c}^{C}\right\}_{\omega^{j}} A_{Q C D C}^{\sin \left(\phi_{2 h}-\phi_{s}\right)}\left(\left\langle x_{C}\right\rangle\right)\right) \\
& =\tilde{\alpha}_{c}^{j}\left(1+A_{P G F}\left\{\beta^{G}\right\}_{\omega^{j}}+A_{L P}\left\{\beta^{L}\right\}_{\omega^{j}}+A_{Q C D C}\left\{\beta^{C}\right\}_{\omega^{j}}\right) .
\end{aligned}
$$

\section{References}

[1] The COMPASS Collaboration, C. Adolph , Phys. Lett. B 718 (2013) 922-930.

[2] The COMPASS Collaboration, C. Adolph et al., Phys. Rev. D 87 (2013) 052018.

[3] Marcin Stolarski on behalf of the COMPASS Collaboration, PoS (DIS2014) 211.

[4] A. Bacchetta et al., JHEP (2007) 02:093.

[5] The COMPASS Collaboration, C. Adolph et al., Phys. Lett. B 692 (2010) 240-246.

[6] D. Rumelhart, G. Hinton, R. Williams, Nature 323 (6088): 533-536. 